\title{
Chimera states in a Duffing oscillators chain coupled to nearest neighbors
}

\author{
M. G. Clerc, ${ }^{1}$ S. Coulibaly, ${ }^{2}$ M. A. Ferré, ${ }^{1}$ and R. G. Rojas ${ }^{3}$ \\ ${ }^{1}$ Departamento de Física and Millennium Institute for Research in Optics, Facultad de Ciencias Físicas y \\ Matemáticas, Universidad de Chile, Casilla, 487-3 Santiago, Chile \\ ${ }^{2}$ Université de Lille, CNRS, UMR 8523-PhLAM-Physique des Lasers Atomes et Molécules, F-59000 Lille, France \\ ${ }^{3}$ Intituto de Física, Pontificia Universidad Católica de Valparaíso, Casilla, 4059 Valparaíso, Chile
}

(Received 6 February 2018; accepted 31 July 2018; published online 31 August 2018)

\begin{abstract}
Coupled nonlinear oscillators can present complex spatiotemporal behaviors. Here, we report the coexistence of coherent and incoherent domains, called chimera states, in an array of identical Duffing oscillators coupled to their nearest neighbors. The chimera states show a significant variation of amplitude in the desynchronized domain. These intriguing states are observed in the bistability region between a homogeneous state and a spatiotemporal chaotic one. These dynamical behaviors are characterized by their Lyapunov spectra and their global phase coherence order parameter. The local coupling between oscillators prevents one domain from invading the other one. Depending on initial conditions, a family of chimera states appear, organized in a snaking-like diagram. Published by AIP Publishing. https://doi.org/10.1063/1.5025038
\end{abstract}

\begin{abstract}
Nonlocal coupling oscillators present rich spatiotemporal dynamics. Depending on the initial conditions, arrays of these oscillators can show coexistence between a desynchronized and a synchronized domain; these states are denominated chimera states. These states are found in an array of oscillators with different coupling schemes. In this article, we investigate chimera states in an array of Duffing oscillators locally coupled. The average frequency characterizes these chimera states, in the desynchronized domain, and varies from one oscillator to another, while in the synchronized domain all remain the same. A family of chimera states with different sizes is observed depending on the initial conditions. A snaking-like diagram organizes these states. To characterize the level of synchronization and the chaotic nature of chimera states, the phase coherence order parameter and the Lyapunov spectrum were determined.
\end{abstract}

\section{INTRODUCTION}

Driven oscillators have been studied since the dawn of mechanics. During the study of the dynamics of the pendulum, Galileo observed the resonance phenomenon. ${ }^{1}$ However, based on a linear forced oscillator, Euler was the first to explain this phenomenon. ${ }^{2}$ A nonlinear generalization of this resonance is possible through the use behavior of the Duffing oscillator, ${ }^{3}$ which is nothing but a nonlinear oscillator with linear dissipation and a harmonic forcing. The Duffing oscillator is known to exhibit a rich variety of dynamical behavior such as periodic and chaotic solutions, bistability, and fractal basin boundaries. When Duffing oscillators are coupled to form an extended system, the resulting array can exhibit complex spatiotemporal dynamics. ${ }^{4}$ The study of coupled oscillators is of great interest owing to their wide applicability in physics, chemistry, and biology (see Ref. 3 and references therein). Likewise, coupled oscillators under the influence of injection and dissipation of energy exhibit a rich spatiotemporal dynamics. Synchronization, defects and/or phase turbulence, defect-mediated turbulence, spatiotemporal intermittency, and coexisting coherent and incoherent states are some of the observed phenomena. ${ }^{5-7}$ In the past decade, a phenomenon that has received a great deal of attention is spatiotemporal patterns in which an array of identical oscillators splits into two domains: one coherent and phase locked and the other incoherent and desynchronized. ${ }^{8}$ This dynamical behavior is referred to as chimera states. The first reports of these intriguing behavior were conducted on an array of oscillators with weak nonlocal ${ }^{9}$ and global coupling schemes. ${ }^{10}$ Chimera states have been studied in a variety of models such as phase oscillators, ${ }^{11-14}$ coupled map lattices, ${ }^{15-18}$ networks, ${ }^{19-21}$ quantum systems, ${ }^{22}$ metamaterials, ${ }^{23}$ neuron models, ${ }^{24,25}$ and delayed systems, ${ }^{26,27}$ and chimera-like states in social systems have been reported. ${ }^{28}$ Experimentally, chimera states have been reported in a chemical system, ${ }^{29}$ optoelectronic oscillators, ${ }^{30,31}$ and a mechanical oscillator network. ${ }^{32}$ Recently, it has been established that chimera-like and chimera states can occur in systems with local (nearest neighbors) $)^{33-36}$ or global coupling. ${ }^{37-39}$

The aim of this paper is to investigate the formation of chimera states in an array of Duffing oscillators chain coupled to their nearest neighbors. In a range of parameters, depending on the initial condition, this model exhibits a coexistence between a synchronized domain and a desynchronized one. The desynchronized domain corresponds to a spatiotemporal chaotic solution. In this region of parameters, we observe chimera states (see Fig. 1). The local coupling between oscillators is to prevent one state from invading the other one. This effect is due to the fact that the coupling induces an effective potential over the dynamics of the interface between domains, the Peierls-Nabarro potential. ${ }^{45,46}$ The average frequency in the desynchronized domains varies discontinuously, such as in chimera states with global coupling. ${ }^{37}$ The global phase coherence order parameter oscillates, showing a behavior such as breathing chimera states. ${ }^{42}$ Depending on the 
(a)

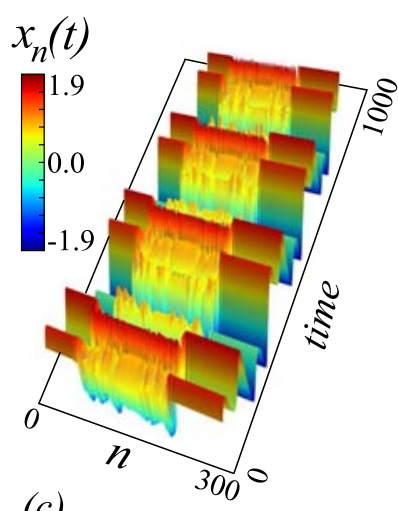

(c)

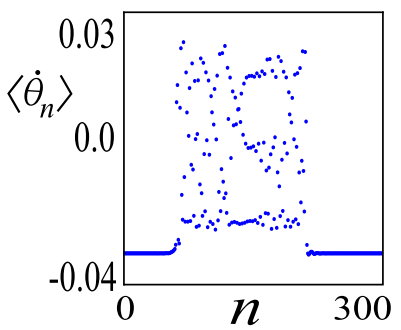

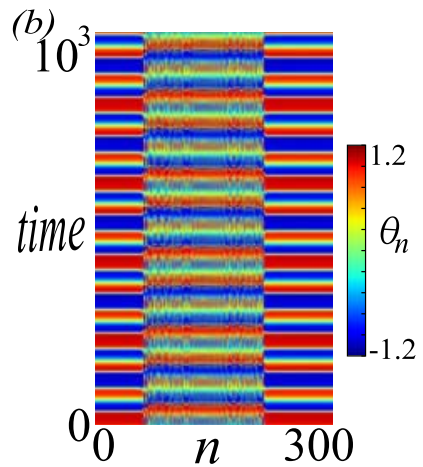

(d)

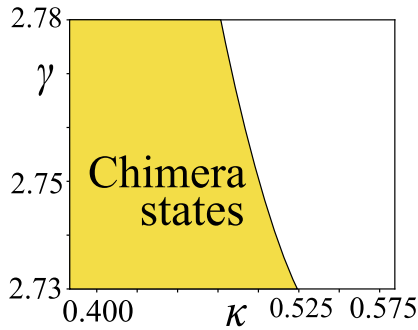

FIG. 1. Chimera state of the Duffing oscillators chain, Eq. (1), by $\alpha=0.4$, $\gamma=2.75, \omega=0.7, \mu=0.1, \kappa=0.42$, and $N=300$. (a) Spatiotemporal diagram of the coupled Duffing oscillators chain. Each oscillator is depicted by $x_{n}(t)$. The colors account for the values of the displacement $x_{n}(t)$. (b) Spatiotemporal evolution of the phase $\theta_{n}(t)=\arctan \left(\dot{x}_{n} / x_{n}\right)$. (c) Temporal average frequency $\left\langle\dot{\theta}_{n}\right\rangle=\int_{0}^{T} \dot{\theta}_{n}(t) d t / T$. All the previous graphics indicate the clear separation between synchronized and desynchronized domains in the coupled Duffing oscillators chain. (d) The phase diagram in the space of $\kappa$ and $\gamma$ parameters.

size of the desynchronized domain, a family of chimera states appear, organized in a snaking-like diagram. This kind of structure in the bifurcation diagram resembles the homoclinicsnaking bifurcation. ${ }^{40,41}$

\section{DUFFING OSCILLATORS CHAIN COUPLED TO NEAREST NEIGHBORS}

Let us introduce a Duffing oscillators chain coupled to nearest neighbors

$$
\begin{aligned}
\ddot{x}_{n}(t)= & -x_{n}+\alpha x_{n}^{3}-x_{n}^{5}-\mu \dot{x}_{n}+\gamma \cos \omega t \\
& +\kappa\left(x_{n+1}-2 x_{n}+x_{n-1}\right),
\end{aligned}
$$

where $x_{n}(t)$ accounts for the displacement of the $n$-oscillator with a unitary natural frequency, $n=\{1,2, \ldots, N\}$ with $\mathrm{N}$ being the total number of oscillators, the nonlinear terms characterize the stiffness, and $\mu$ stands for the damping coefficient. The term proportional to $\gamma$ accounts for the external forcing with amplitude and frequency being $\gamma$ and $\omega$, respectively. $\kappa$ is the coupling parameter. Note that coupling between the oscillators is modeled by means of a linear spring of elastic constant $\kappa$. Figure 1 shows a typical chimera state founded in Eq. (1). The color bar depicts the value of each oscillator displacement in a given time. Figure 1(b) depicts the spatiotemporal dynamics of the oscillator phase $\theta_{n} \equiv \arctan \left(\dot{x}_{n} / x_{n}\right)$. The spatiotemporal diagram of the oscillator displacement and phase indicate a clear separation between synchronized and desynchronized domains. Let us introduce the average frequency $\left\langle\dot{\theta}_{n}\right\rangle \equiv \frac{1}{T} \int_{0}^{T} \dot{\theta}_{n}(t) d t$, where $T$ is chosen several times larger of the forcing period, $T \gg 2 \pi / \omega$. The phase diagram in the space of $\kappa$ and $\gamma$ parameters is depicted in Fig. 1(d).

\section{LOCAL DYNAMICS OF THE DUFFING OSCILLATOR}

To figure out the existence of the chimera states in model Eq. (1), it is necessary to understand the dynamics of an individual oscillator. Neglecting the effects of coupling, i.e., $\kappa=0$ and fixing the value of the nonlinear stiffness and the dissipation parameters ( $\alpha=0.4$ and $\mu=0.1$ ), we will study the dynamics of the forced nonlinear oscillator Eq. (1). This oscillator is called the cubic-quintic Duffing oscillator. Using as order parameter the maximum value reached by the periodic solution $x_{n}(t)$, defined as $X_{\max }$, we obtain numerically two bifurcation diagrams for this forced nonlinear oscillator. In the first bifurcation diagram, the control parameter is the forcing frequency $\omega$ [cf. Fig. 2(a)]. In the second one, the control parameter is the strength of the forcing $\gamma$. Figure 2(b) shows these bifurcation diagrams. The insets account for the phase portraits of each periodical solution labeled in the bifurcation diagrams. To identify all periodic solutions in both bifurcation diagrams, we have considered 200 different initial

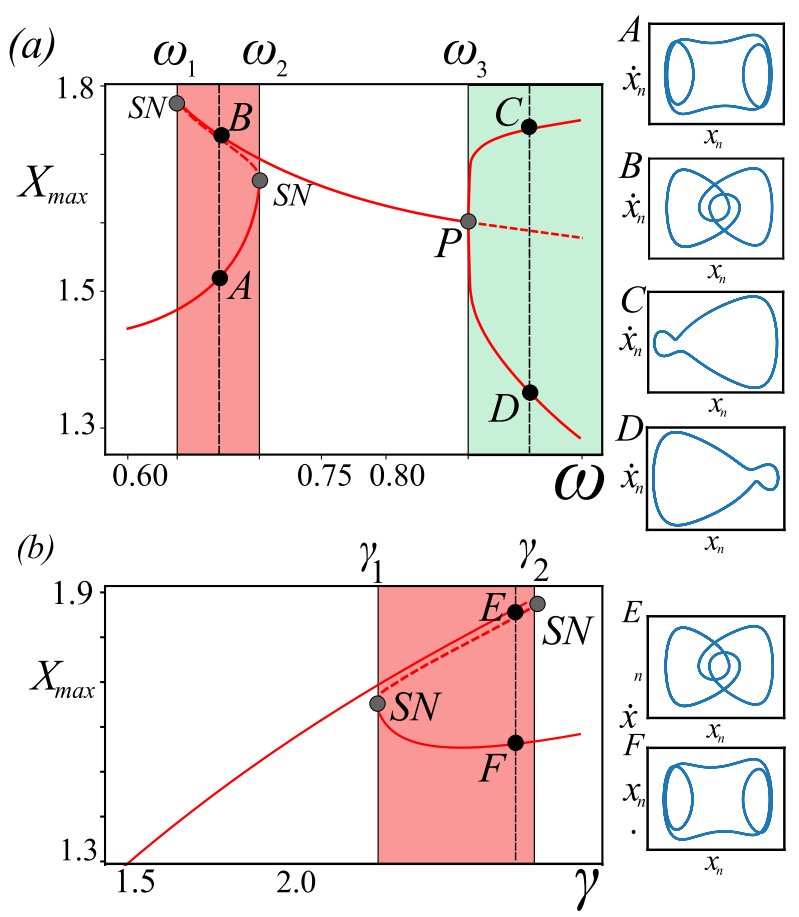

FIG. 2. Bifurcation diagram of the Duffing oscillator Eq. (1) with $\kappa=0$, $\alpha=0.4$, and $\mu=0.1$ considering as a control parameter (a) forcing frequency $\omega$ with $\gamma=2.75$ and (b) the strength of the forcing $\gamma$ with $\omega=0.7$. The order parameter $X_{\max }$ accounts for the maximum value reached by the periodic oscillation. The solid and dashed curves stand for stable and unstable solutions, respectively. Insets illustrate the portrait phase of the different solutions. In the top bifurcation diagram, the system exhibits bistability in two different regions. The darker (reddish) area, between $\omega_{1}=0.655$ and $\omega_{2}=0.855$, accounts for the subcritical bifurcation. The lightest painted area (greenish) accounts for the pitchfork bifurcation since $\omega_{3}=0.863$. In the bottom bifurcation diagram, only one bistability region is found between $\gamma_{1}=2.730$ and $\gamma_{2}=3.518$. The appearance of this bistability region corresponds to a subcritical bifurcation. 
conditions uniformly distributed on the straight line between the points $\left(x_{0}, \dot{x}_{0}\right)=(2.0,2.0)$ and $\left(x_{1}, \dot{x}_{1}\right)=(-2.0,-2.0)$. Later the trajectories evolve using a fourth-order Runge-Kutta method with a temporal discretization $d t=0.01$. In Fig. 2(a), for small frequencies, one only observes a single periodic solution as equilibrium. We call this solution as the local lower oscillation. This solution has a morphology similar to that illustrated by the periodic curve labeled by the letter A. Increasing the value of the frequency forcing until $\omega_{1}=0.639$, a second stable periodical solution appears by a saddle-node bifurcation. We call this solution as the local upper oscillation. The symbol $S N$ represents the saddle-node bifurcation. The morphology of the local upper oscillation is similar to that illustrated by the curve labeled by the letter B. This two stable periodic solutions coexist until $\omega_{2}=0.704$, where the local lower oscillation disappears by a saddle-node bifurcation. Hence, in this region of the parameter space; the system exhibits a subcritical bifurcation. The local upper oscillation persists until $\omega_{3}=0.863$, where two new asymmetric periodical solutions, C-solution and D-solution, appear by pitchfork bifurcation. This instability is represented by $P$ point. Hence, as a function of the forcing frequency, the Duffing oscillator Eq. (1) exhibits a rich variety of bifurcations allowing the coexistence of different periodic solutions. In Fig. 2(b), for small values of $\gamma$, an only single periodical solution exists, the local upper oscillation. Increasing the strength of the forcing and reaching the value $\gamma_{1}=1.436$, a second periodical solution arises by saddle-node bifurcation. This solution corresponds to the local lower oscillation. When $\gamma$ is larger as $\gamma_{2}=3.490$, the local lower oscillation persists, but the local upper oscillation ceases to exist by a saddle-node bifurcation. The local upper solution and the local lower solution show a phase portrait similar to the solutions depicted by the letters E and F, respectively. Therefore, the system exhibits a subcritical bifurcation. Only one region of coexistence between periodic solutions is identified in the bifurcation diagrams previously described. It is in these regions of coexistence one expects that when one extends the system spatially complex domains emerge between different states. For the sake of simplicity of the bifurcation diagram, the strength of the forcing is chosen as the control parameter in this study.

\section{CHIMERA STATES IN A DUFFING OSCILLATORS CHAIN}

When the Duffing oscillators are coupled to nearest neighbors by means of linear springs, depending on the parameter $\kappa$, periodic oscillations can be synchronized or desynchronized. ${ }^{6}$ When the strength of the coupling is large, one expects to observe synchronization of the oscillators in the chain. Likewise, when the strength of the forcing is (a)

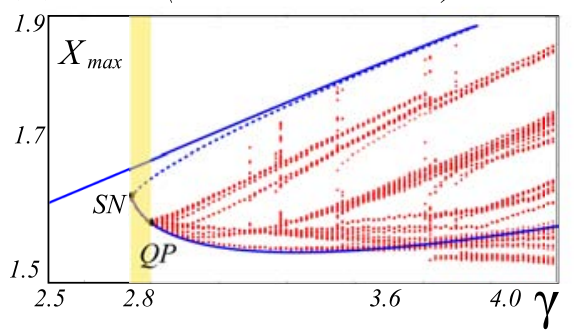

(c) (Lower oscillation)
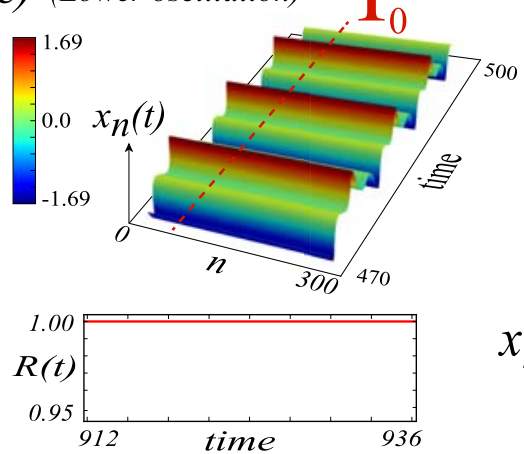

(e)

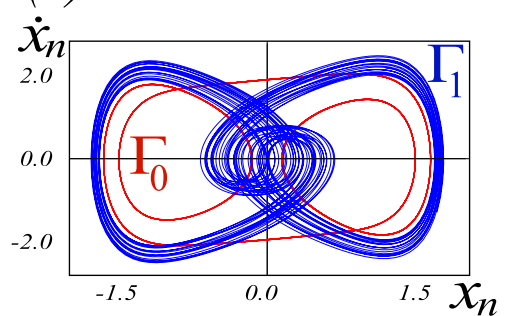

(b)

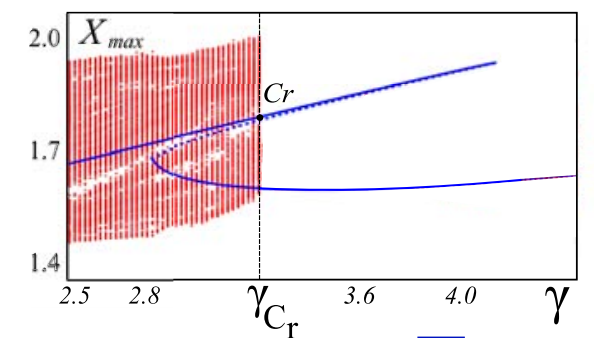

(d)
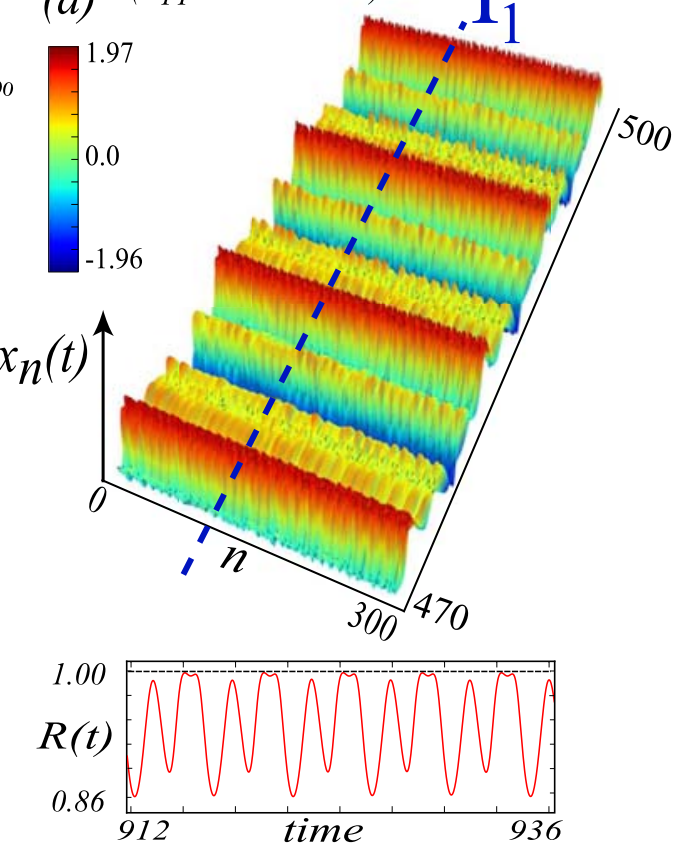

FIG. 3. Duffing oscillators chain Eq. (1). Bifurcation diagram of the lower (a) and the upper extended oscillation (b) of the Duffing oscillator chain coupled to nearest neighbors Eq. (1) with $\kappa=0.42, \alpha=0.4, \mu=$ 0.1 , and $\omega=0.7$. Solid and dashed (blue) curves account for the bifurcation diagram of one isolated oscillator. Dots account for the different maximum values, $X_{\max }$, exhibited in a given arbitrary oscillator when time evolves. The shaded area accounts for the bistability region between a uniform and chaotic extended oscillation. Spatiotemporal diagrams of the coherent (c) and incoherent (d) extended oscillation for $\gamma=2.75$, which correspond, respectively, to the lower and the upper branch. The insets correspond to the global phase coherence order parameter $\mathrm{R}(t)$ as a function of time. (e) Phase portrait of an oscillator in the coherent $\left(\Gamma_{0}\right)$ and incoherent oscillation $\left(\Gamma_{1}\right)$. 
decreased, one expects to observe desynchronization of the oscillators, which is characterized by complex spatiotemporal behaviors. This type of dynamics can be classified into three types: quasi-periodic, chaotic, or spatiotemporal chaotic. Figure 3 shows the bifurcation diagram of the lower (a) and the upper (b) extended oscillations. To obtain the lower (upper) extended oscillation, we consider as an initial condition the local lower oscillation (local upper oscillation) for all the oscillators in model Eq. (1) with a small random perturbation. Figure 3 shows the maximum values, $X_{\max }$, of all the oscillators. Hence, the synchronized lower oscillation appears by a saddle-node bifurcation. The saddle-node bifurcation is highlighted by the point $S N$. By increasing the strength of the forcing, this periodic homogeneous solution becomes quasiperiodic. $Q P$ designates this bifurcation. Further increasing the strength of the forcing, this oscillation becomes chaotic by means of an extended quasi-periodicity route. ${ }^{43}$ Hence, there is a narrow region of the parameter space where the lower extended oscillation is synchronized. A shaded zone emphasizes this region between the saddle-node bifurcation and the quasi-periodic bifurcation. Figure 3(c) depicts the typical spatiotemporal evolution of this lower extended oscillation in the synchronized region. To characterize the synchronization, let us introduce the following global phase coherence order parameter proposed by Kuramoto: ${ }^{5}$

$$
R(t)=\frac{1}{N}\left|\sum_{i=1}^{N} e^{i \theta_{i}(t)}\right|,
$$

where the phase of each oscillator is defined as before. When the oscillators are synchronized, this parameter is one $(R=1)$; if they are desynchronized, this parameter is less than one $(R<1)$. The inset in Fig. 3(c) shows that the oscillators are permanently synchronized.

Analogously, we have performed a similar analysis for the upper extended oscillation. Figure 3(b) summarizes the findings found. For small forcing strength, the upper oscillation is of the spatiotemporal chaotic nature. The spatiotemporal evolution of this oscillation is shown in Fig. 3(d). The synchronization parameter oscillates in time, showing moments of synchronization between the oscillators. In our system, this behavior is permanent. Long numerical simulations have been carried out, and the dynamics of the system does not change before the critical value $\gamma_{C_{r}}$. When $\gamma>\gamma_{C_{r}}$, the spatiotemporal chaos abruptly disappears. This instability corresponds to an external crisis. ${ }^{44}$ Figure 3 (b) emphasizes the crisis phenomenon by $\mathrm{Cr}$. Subsequent to this crisis instability, the spatiotemporal chaos of upper oscillation is replaced

\section{(a)}

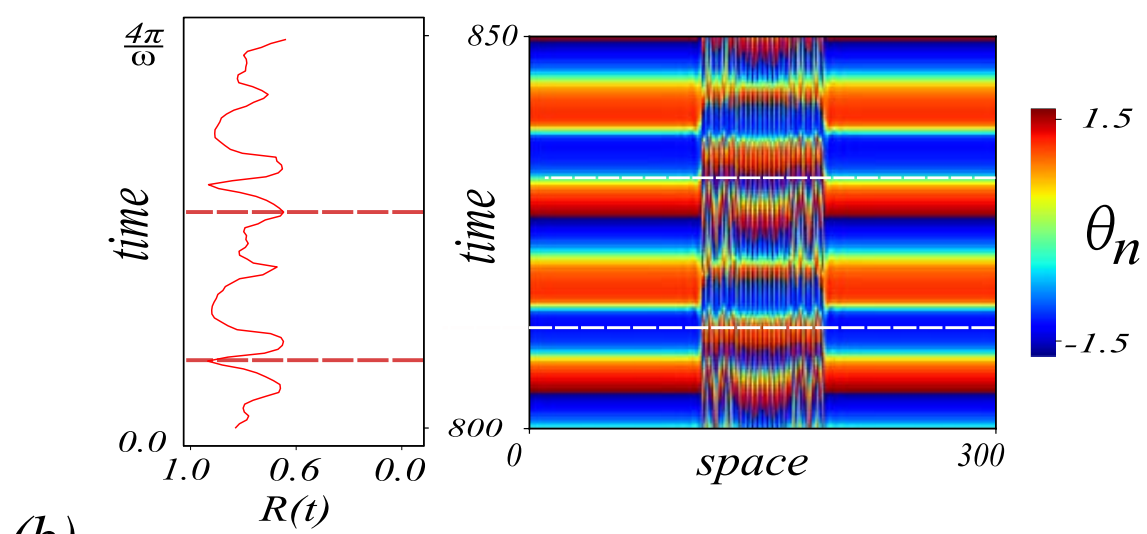

(b)
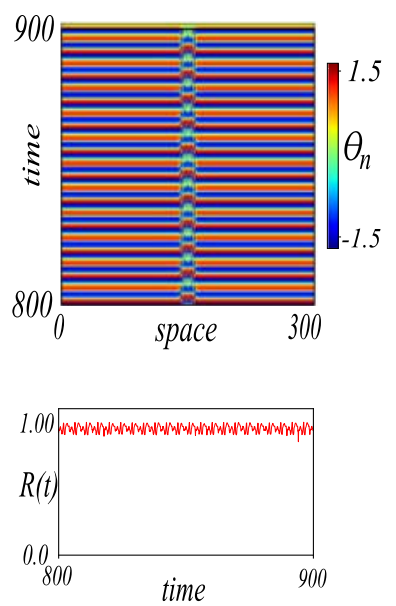
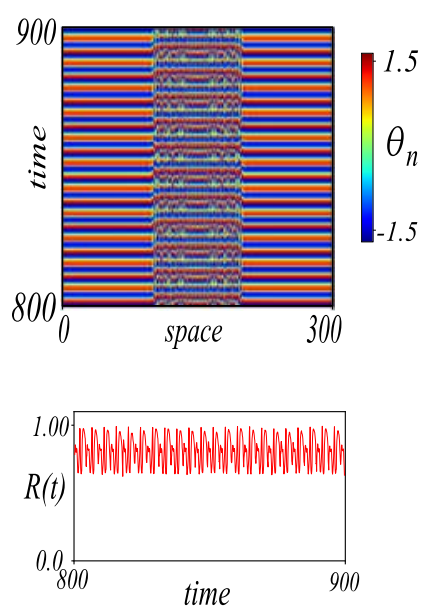
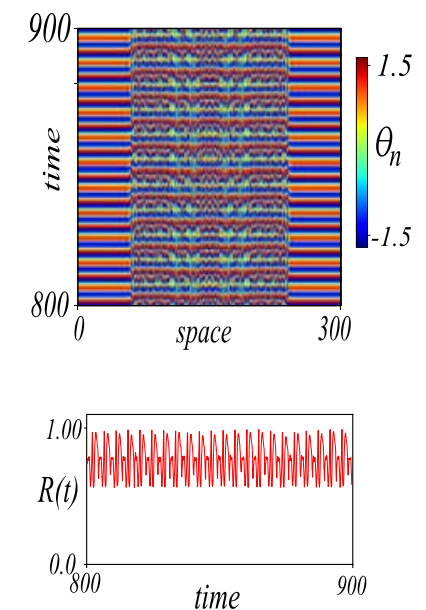

FIG. 4. Temporal evolution for the global phase coherence order parameter $\mathrm{R}(t)$ and spatiotemporal diagrams for chimera states of the Duffing oscillators chain Eq. (1) with $\kappa=0.42, \alpha=0.4$, $\mu=0.1$, and $\omega=0.7$. (a) Temporal evolution of $R(t)$ and spatiotemporal diagram of chimera state in a few periods $4 \pi / \omega$. Segmented horizontal lines account for extreme values of $R(t)$ in a period $T_{0}=2 \pi / \omega$. The colors account for the value of the displacement of the oscillators $x_{n}(t)$. (b) Temporal evolution of $R(t)$ and spatiotemporal diagrams of chimera states with different sizes. 
by the lower extended chaotic oscillation. Figure 3(e) shows the orbits in the phase portrait obtained when one considers the evolution of an individual oscillator of the chain for a synchronized oscillation $\left(\Gamma_{0}\right)$ and a spatiotemporal chaotic one $\left(\Gamma_{1}\right)$.

From the bifurcation diagrams of the upper and the lower extended oscillation, we can infer that the Duffing oscillators chain, Eq. (1), has a bistability region between a chaotic oscillation state (upper extended oscillation) and a synchronized uniform oscillation (lower extended oscillation). The shadow zone in Fig. 3(a) illustrates this bistability region. Hence, in this region, one expects to observe chimera states, since the coupling prevents the most favorable state and invades the less favorable one. ${ }^{34}$ The coupling induces a periodic potential over the dynamics of the interface between domains. This periodic potential is well known as the Peierls-Nabarro potential. ${ }^{45,46}$ This potential prevents the interface propagation. The typical chimera state found in the Duffing oscillators chain coupled to nearest neighbors model Eq. (1) is shown in Fig. 1. The synchronized (desynchronized) domain corresponds to an oscillation close to the lower (upper) extended oscillation. To obtain a chimera state, one must consider an initial condition that connects spatially the lower and the upper extended oscillation with a small random perturbation. Coexistence between a complex localized dynamics and periodical behavior is observed. Figure 1(b) shows the spatiotemporal evolution of the phase in the chimera state. The synchronized domain shows a homogeneous behavior in space and periodical behavior in time. The desynchronized domain shows a complex spatiotemporal behavior. The coexistence of coherent and incoherent states is the main characteristic of the chimera states. The rigorous characterization of the incoherent dynamical behavior will be discussed later. The average frequency $\left\langle\dot{\theta}_{n}\right\rangle$ was measured for each oscillator and is shown in Fig. 1(c). Note that, in the synchronized domain, $\left\langle\dot{\theta}_{n}\right\rangle$ is equal; however, in the desynchronized domain, a discontinuous variation is observed. This feature resembles the case of chimera states with global coupling, ${ }^{37}$ where the average frequency has a similar shape as shown in Fig. 1(c). However, in the case of chimera states in phase-oscillators with a nonlocal coupling scheme, the average frequency changes smoothly in the desynchronized domain. ${ }^{8,9}$ The transition between a discontinuous and continuous variation of average frequency in the desynchronized domain is still an open question. To characterize the complexity of chimera solutions, Fig. 4 shows the temporal evolution of the global phase coherence order parameter $\mathrm{R}(t)$ for chimera states. During a period of forcing, the global phase coherence order parameter exhibits complex fluctuations [cf. Fig. 4(a)], showing a behavior known as breathing chimera states. ${ }^{42}$ Hence, in certain moments, the chimera state alternates between being more or less coherent.

A rigorous way of characterizing the complexity of chimera states is through the use of the Lyapunov spectrum. ${ }^{34,35}$ This spectrum provides information about permanent dynamic with exponential sensitivity to initial conditions. ${ }^{47}$ When the largest Lyapunov exponent is negative, the system has a stationary equilibrium, such as uniform or pattern states. This scenario changes when the largest Lyapunov exponent is positive, the system exhibits chaotic dynamics but not necessarily spatiotemporal chaos. Spatiotemporal chaos has a Lyapunov spectrum with a continuous set of positive values. In opposition, low dimensional chaos possesses a Lyapunov spectrum with a discrete set of positive values. $\lambda_{i}$ accounts for the Lyapunov exponents, where $i$ indexes the variables of the system $(i=1, \ldots, 2 N)$. The Lyapunov exponents are sorted in a decreasing manner. ${ }^{47}$ Using the strategy proposed in Ref. 48, we have computed the Lyapunov spectrum numerically for different chimera states. Figure 5(a) shows the Lyapunov spectra for different states of Eq. (1). Note that the spectrum of the synchronized oscillation is flat for the dominant Lyapunov exponents. From this figure, we can conclude that the larger the chimera state, the larger the number of Lyapunov positive exponents. That is, (a)

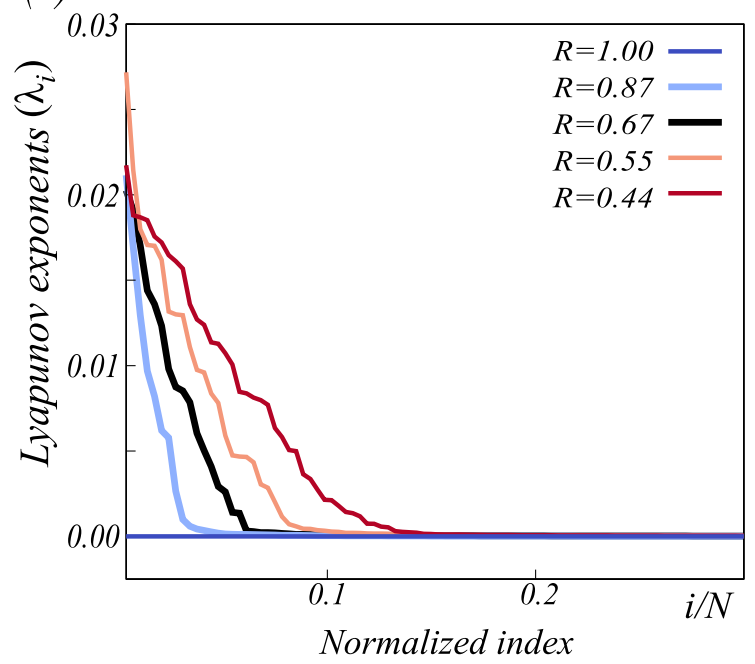

(b)

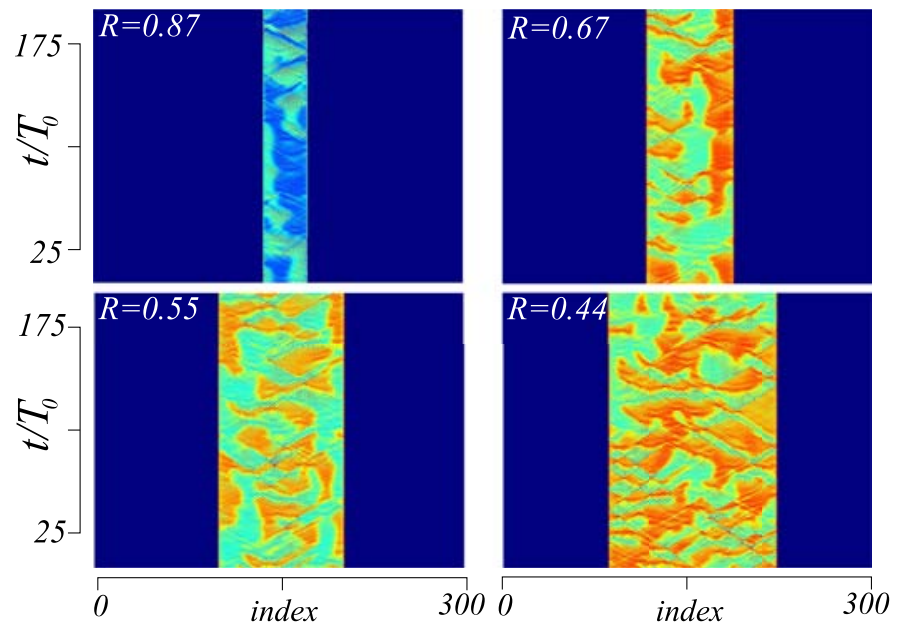

FIG. 5. Family of chimera solutions of the Duffing oscillators chain Eq. (1) with $\alpha=2.0, \gamma=7.85, \mu=0.05, \omega=0.985$, and $\kappa=0.39$. (a) Lyapunov spectra of different chimera states. (b) Stroboscopic spatiotemporal diagram of different chimera-like states using as observation period $T_{0}=2 \pi / \omega$. $R$ accounts for the averaging synchronization order parameter formula (2) with $T=200 T_{0}$. 
the dynamics exhibited by the chimera state are wider and more complex. Likewise, we note that the largest Lyapunov exponent of each spectrum is of the same order. Figure 5(b) shows the spatiotemporal stroboscopic diagram for chimera states of different sizes. These diagrams correspond to a generalization of the Poincaré maps, and the profile of the system was captured for each period of forcing $T_{0}=2 \pi / \omega$. Notice that the complexity of the dynamics grows with the size of the chimera state. Since the forcing frequency $\omega$ is close to the natural frequency of the nonlinear oscillator $\omega_{0}=1$, the oscillatory behavior of the synchronized domain can be ignored through the use of stroboscopic analysis [cf. Fig. 5(b)]. In this region of parameters, the bistability region operates for higher gamma values. For the value of the parameters in Fig. 5, the same kind of periodical solutions as Fig. 2 is founded.

\section{MULTISTABILITY AND BIFURCATION DIAGRAM OF CHIMERA STATES}

Due to the pinning effect generated by the local coupling between the oscillators, ${ }^{45}$ one expects to observe a family of chimeras. Consequently, our chimeras present a snakinglike bifurcation diagram, ${ }^{40,41}$ that is, these states appear and disappear by a sequence of saddle-node bifurcations, which occur all around a given value of the nonlinearity parameter in both extremes of the coexistence region. Hence, in a range of parameters, one observes the coexistence of chimera solutions. To characterize this bifurcation diagram, we introduce the halfway area of chimera states, $\mathcal{A}\left(t^{*}\right) \equiv \sum_{i} x_{i}\left(t^{*}\right)$. This area is calculated at the moment that the incoherent state presents its greatest amplitude $t=t^{*}$ in a period of forcing. Introducing the area of chimera state

$$
\mathcal{A} \equiv \sum_{i=1}^{M} \mathcal{A}\left(t_{i}^{*}\right) / M
$$

which correspond to the average of the halfway area after a large number of period $(M \gg 1)$. The area of chimera state $\mathcal{A}$ is used as an order parameter. Figure 6 shows the average area $\mathcal{A}$ as a function of the strength of the forcing $\gamma$. Note that the chimera states are in a range bounded between two amplitudes $\left\{\gamma_{-}, \gamma_{+}\right\}$. Outside this region, for the smaller (greater) strengths of the forcing, the incoherent (coherent) state invades the coherent (incoherent) one. Hence, a completely extended state is observed in this region. Namely, the upper and lower branches of the snaking-like bifurcation diagram correspond to the entire incoherent and coherent domains, respectively. Multistability of chimera states has been reported in coupled oscillators, ${ }^{34}$ coupled waveguides, ${ }^{35}$ and superconducting quantum interference device oscillators. ${ }^{36}$ Also, we can measure the global phase

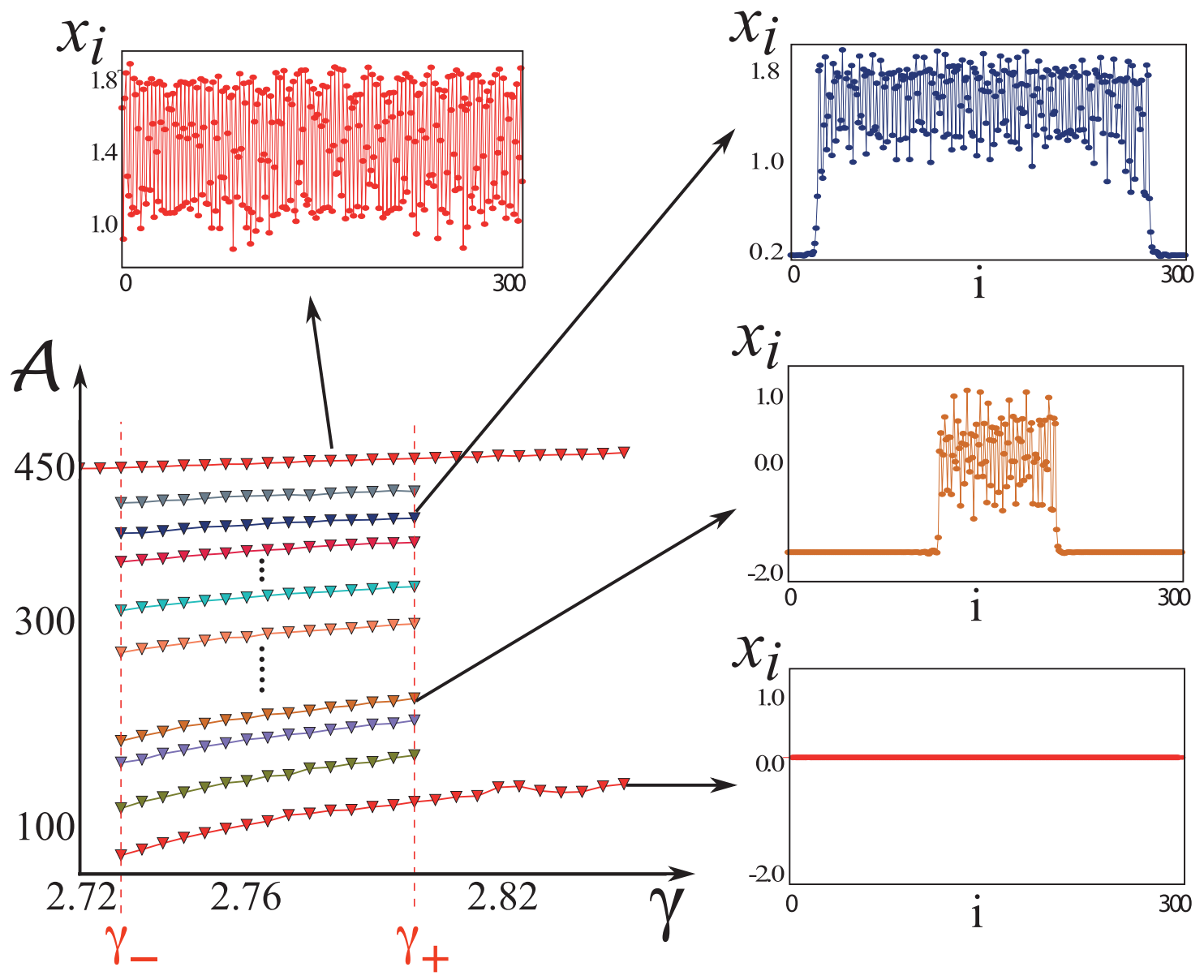

FIG. 6. Snaking-like bifurcation diagram of chimera states of the Duffing oscillators chain Eq. (1) with $\kappa=0.42, \alpha=0.4, \mu=0.1$, and $\omega=0.7$. Area of chimera states $\mathcal{A}$, formula (3), as a function of the strength of the forcing $\gamma$. The triangular symbols $(\nabla)$ account for the value of the area of the chimera-like state obtained numerically. The insets illustrate the state at a given time. 
coherence order parameter $R(t)$ for the family of chimera solutions with different sizes. We found that as the chimera is larger, the fluctuation range of $R(t)$ is more significant but still oscillates in a complex manner, as illustrated in Fig. 4(b).

\section{CONCLUSION}

In conclusion, we have shown the coexistence of coherent and incoherent states, chimera states, in a simple Duffing oscillators chain coupled to nearest neighbors. These intriguing states are observed in the bistability region between a uniform oscillation and a spatiotemporal chaotic state. To characterize the chimera states rigorously, we have computed their Lyapunov spectra. Depending on initial conditions, a family of chimera states can appear and disappear, following a snaking-like bifurcation diagram. Increasing the strength of the forcing, the coherent domains (synchronized oscillation) become unstable, and a spatiotemporal chaotic behavior emerges. Then, in this region of parameters, the system exhibits the coexistence of two incoherent states. The study of these states is in progress.

The Duffing oscillator is a paradigmatic model of the forced nonlinear oscillators. Hence, one expects that forced and coupled oscillators in several physical systems should exhibit chimera states. Recently, the Duffing model has been used to describe nano-electromechanical ${ }^{49}$ and nano-electromechanical oscillators. ${ }^{50}$ Coupled nanoelectromechanical membranes could be an ideal system to study chimeric states.

\section{ACKNOWLEDGMENTS}

M.G.C. and M.A.F. are thankful for the financial support of CONICYT-USA, Project No. PII20150011 and Millenium Institute for Research in Optics (MIRO). S.C. and R.G.R. are grateful for the financial support of ECOS-CONICYT (No. C15E06).

${ }^{1}$ A. Frova and M. Marenzana, Thus Spoke Galileo: The Great Scientist's Ideas and Their Relevance to the Present Day (Oxford University Press, 2006).

${ }^{2}$ L. Euler, "De novo genere oscillationum," Comment. Acad. Sc. Petrop. 11, $128(1750)$.

${ }^{3}$ I. Kovacic and M. J. Brennan, The Duffing Equation: Nonlinear Oscillators and their Behaviour (John Wiley and Sons, 2011).

${ }^{4}$ D. K. Umberger, C. Grebogi, E. Ott, and B. Afeyan, Phys. Rev. A 39, 4835 (1989).

${ }^{5}$ Y. Kuramoto, Chemical Oscillations, Waves, and Turbulence (Courier Corporation, 2003).

${ }^{6}$ A. Pikovsky, M. Rosenblum, J. Kurths, and R. C. Hilborn, Synchronisation: A Universal Concept in Nonlinear Sciences (Cambridge University Press, 2002).

${ }^{7} \mathrm{~K}$. Kaneko and I. Tsuda, Chaos and Beyond: A Constructive Approach with Applications in Life Sciences (Springer, 1996).

${ }^{8}$ D. M. Abrams and S. H. Strogatz, Phys. Rev. Lett. 93(17), 174102 (2004).

${ }^{9}$ Y. Kuramoto and D. Battogtokh, Nonlinear Phenom. Complex Syst. 5, 380 (2002).

${ }^{10}$ K. Kaneko, Physica D 41, 137 (1990).

${ }^{11}$ G. C. Sethia, A. Sen, and G. L. Johnston, Phys. Rev. E 88, 042917 (2013).
${ }^{12}$ O. E. Omel'chenko, Nonlinearity 26(9), 2469 (2013).

${ }^{13}$ L. Smirnov, G. Osipov, and A. Pikovsky, J. Phys. A 50(8), 08LT01 (2017).

${ }^{14}$ E. A. Martens, M. J.Panaggio, and D. M. Abrams, New J. Phys. 18(2), 022002 (2016).

${ }^{15}$ D. Dudkowski, Y. Maistrenko, and T. Kapitaniak, Phys. Rev. E 90, 032920 (2014).

${ }^{16}$ R. Gopal, V. K.Chandrasekar, A. Venkatesan, and M. Lakshmanan, Phys. Rev. E 89, 052914 (2014).

${ }^{17}$ E. Schöll, Eur. Phys. J. Spec. Top. 225, 891-919 (2016).

${ }^{18}$ E. Omel'chenko, Y. L.Maistrenko, and P. A. Tass, Phys. Rev. Lett. 100(4), 044105 (2008).

${ }^{19}$ E. A. Martens, Phys. Rev. E 82, 016216 (2010).

${ }^{20}$ I. Omelchenko, E. Omel'chenko, A. Zakharova, M. Wolfrum, and E. Schöll, Phys. Rev. Lett. 116, 114101 (2016).

${ }^{21}$ L. Tumash, A. Zakharova, J. Lehnert, W. Just, and E. Schöll, Europhys. Lett. 117, 20001 (2017).

${ }^{22}$ V. M. Bastidas, I. Omelchenko, A. Zakharova, E. Schöll, and T. Brandes, Phys. Rev. E 92, 062924 (2015).

${ }^{23}$ N. Lazarides, G. Neofotistos, and G. P. Tsironis, Phys. Rev. B 91, 054303 (2015).

${ }^{24}$ J. Hizanidis, V. G. Kanas, A. Bezerianos, and T. Bountis, in 2014 13th International Conference on Control Automation Robotics and Vision (ICARCV) (IEEE, 2014, December), pp. 243-246.

${ }^{25}$ M. S. Santos, J. D. Szezech, F. S. Borges, K. C. Iarosz, I. L. Caldas, A. M. Batista, R. L. Viana, and J. Kurths, Chaos Solitons Fractals 101, 86-91 (2017).

${ }^{26}$ L. Larger, B. Penkovsky, and Y. Maistrenko, Phys. Rev. Lett. 111, 054103 (2013).

${ }^{27}$ B. K. Bera and D. Ghosh, Phys. Rev. E 93, 052223 (2016).

${ }^{28}$ J. C. Gonzalez-Avella, M. G. Cosenza, and M. San Miguel, Physica A 399, 24 (2014).

${ }^{29}$ M. R. Tinsley, S. Nkomo, and K. Showalter, Nat. Phys. 8, 662-665 (2012).

${ }^{30}$ A. M. Hagerstrom, T. E. Murphy, R. Roy, P. Hövel, I. Omelchenko, and E. Schöll, Nat. Phys. 8, 658-661 (2012).

${ }^{31}$ J. D. Hart, K. Bansal, T. E. Murphy, and R. Roy, Chaos 26, 094801 (2016).

${ }^{32}$ E. A. Martens, S. Thutupalli, A. Fourrière, and O. Hallatschek, Proc. Natl. Acad. Sci. 110(26), 10563-10567 (2013).

${ }^{33}$ C. R. Laing, Phys. Rev. E 92(5), 050904 (2015).

${ }^{34}$ M. G. Clerc, S. Coulibaly, M. A. Ferré, M. A. García-Ñustes, and R. G. Rojas, Phys. Rev. E 93, 052204 (2016).

${ }^{35}$ M. G. Clerc, M. A. Ferré, S. Coulibaly, R. G. Rojas, and M. Tlidi, Opt. Lett. 42, 2906-2909 (2017).

${ }^{36}$ J. Hizanidis, N. Lazarides, and G. P. Tsironis, Phys. Rev. E 94, 032219 (2016).

${ }^{37}$ G. C. Sethia and A. Sen, Phys. Rev. Lett. 112, 144101 (2014).

${ }^{38}$ A. Yeldesbay, A. Pikovsky, and M. Rosenblum, Phys. Rev. Lett. 112, 144103 (2014).

${ }^{39}$ C. R. Hens, A. Mishra, P. K. Roy, A. Sen, and S. K. Dana, Pramana 84, 229-235 (2015).

${ }^{40}$ P. D. Woods and A. R. Champneys, Physica D 129, 147-170 (1999).

${ }^{41}$ J. Burke and E. Knobloch, Chaos 17, 037102 (2007).

${ }^{42}$ D. M. Abrams, R. Mirollo, S. H. Strogatz, and D. A. Wiley, Phys. Rev. Lett. 101(8), 084103 (2008).

${ }^{43}$ M. G. Clerc and N. Verschueren, Phys. Rev. E 88, 052916 (2013).

${ }^{44}$ E. Ott, Chaos in Dynamical Systems (Cambridge University Press, 2002).

${ }^{45}$ M. G. Clerc, R. G. Elías, and R. G. Rojas, Philos. Trans. Roy. Soc. Lond. Ser. A 369(1935), 412-424 (2011).

${ }^{46}$ O. M. Braun and Y. S. Kivshar, The Frenkel-Kontorova Model: Concepts, Methods, and Applications (Springer, 2013).

${ }^{47}$ A. Pikovsky and A. Politi, Lyapunov Exponents: A Tool to Explore Complex Dynamics (Cambridge University, 2016).

${ }^{48} \mathrm{C}$. h. Skokos, "The Lyapunov characteristic exponents and their computation," Dynamics of Small Solar System Bodies and Exoplanets (Springer, 2010), pp. 63-135.

${ }^{49}$ A. Chowdhury, S. Barbay, M. G. Clerc, I. Robert-Philip, and R. Braive, Phys. Rev. Lett. 119, 234101 (2017).

${ }^{50}$ R. M. C. Mestrom, R. H. B. Fey, J. T. M. Van Beek, K. L. Phan, and H. Nijmeijer, Sens. Actuators A Phys. 142, 306 (2008). 